\title{
Theory and Design of Tunable Full-Mode and Half- Mode Ferrite Waveguide Isolators
}

\author{
Farhan A. Ghaffar ${ }^{1,3}$, Joey R. Bray ${ }^{2}$, Member IEEE, Mohammed Vaseem ${ }^{1}$, Langis Roy ${ }^{3}$, Atif Shamim ${ }^{1}$, Senior \\ Member IEEE \\ ${ }^{1}$ CEMSE Division, King Abdullah University of Science and Technology, KAUST, Thuwal, Saudi Arabia \\ ${ }^{2}$ Department of Electrical and Computer Engineering, Royal Military College, RMC, Kingston, Canada \\ ${ }^{3}$ Faculty of Engineering and Applied Science, Ontario Tech University, UOIT, Oshawa, Canada
}

\begin{abstract}
Ferrite isolators are attractive due to their excellent isolation, low loss, good linearity and high power performance. However, these devices usually operate over a single frequency band. Multiband applications are possible if the isolation bandwidth of an isolator can be tuned. This paper presents a tunable waveguide-based full-mode ferrite isolator as well as a more compact half-mode tunable isolator, both fabricated on yttrium-iron-garnet substrates. For the first time, theory and design guidelines for the center frequency, bandwidth and tuning of the ferrite isolators' unidirectional magnetostatic surface wave (MSW) mode are presented. The proposed theoretical model reveals that the isolation bandwidth can exceed $100 \%$ if the magnetization-to-bias field ratio is higher than 8. While the full-mode design is fabricated using a conventional subtractive technique, the half-mode design is implemented using inkjet printing technology. The full-mode isolator provides a maximum bandwidth of $45 \%$ and a peak isolator figure of merit (IFM) of over 65 dB at $7 \mathrm{GHz}$, whereas the half-mode design has a maximum bandwidth of 59\% and a peak IFM of $76.7 \mathrm{~dB}$ at $7.5 \mathrm{GHz}$. The tuning of the centre frequency is from $7 \mathrm{GHz}$ to $10.7 \mathrm{GHz}$ for the full-mode and from $4.4 \mathrm{GHz}$ to $9.9 \mathrm{GHz}$ for the half-mode design using magnetic field strengths up to $2500 \mathrm{Oe}$ and $2400 \mathrm{Oe}$, respectively. This work demonstrates the versatility of ferrite isolators as tunable mi crowave devices for reconfigurable RF applications.
\end{abstract}

Index Terms - isolator, ferrite, waveguide, isolator figure of merit

\section{INTRODUCTION}

The unidirectional properties of microwave isolators are useful for protecting the output of load-sensitive devices against unwanted reflections. For example, the inclusion of an isolator at the output of an oscillator serves to protect it from loadpulling [1]. Similarly, an isolator may be used at the output of a high power amplifier to prevent load mismatch from affecting its stability and efficiency [2]. Isolators have been realized using various technologies at different integration levels [3] [5]. However, none of these technologies can compare to ferrite based designs in terms of the bandwidth, power and high isolation performance.

Ferrite isolators are based on different nonreciprocal phenomena, such as resonance absorption [6], Faraday rotation [7], and field displacement [8], and have been implemented in different media, including coaxial lines and microstrip. Rectangular waveguide (RWG) isolators have also been demonstrated where low loss and high power handling capability may be required [9]. When a ferrite-filled RWG is biased by a magnetic field normal to its axis, a negative effective permeability permits a magnetostatic surface wave (MSW) mode to propagate in one direction only over a certain range of frequencies, which is dependent on the saturation magnetization of the ferrite material and the applied magnetic field. This property of ferrite has been used to demonstrate many isolator designs [8], [10]-[12].

Manuscript received ....

Corresponding author: Farhan A. Ghaffar (farhan.ghaffar@kaust.edu.sa)

Authors, Farhan A. Ghaffar and Joey R. Bray contributed equally to this manuscript

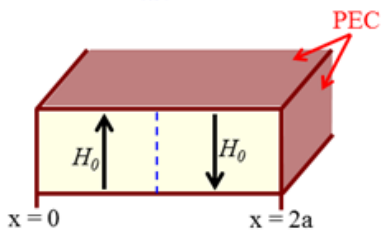

(a)

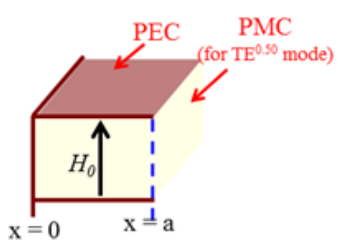

(b)
Fig.1. Ferrite filled waveguide based isolator designs (a) full-mode anti-symmetrically biased (b) half-mode with a single direction of bias

Although ferrite isolators have superior performance, there are only a few designs which show frequency tunability. Some tunable ferrite loaded isolator designs have been presented recently [13]-[15]. Two of the designs are realized by loading a microwave feed line with a biased ferrite material, a configuration that yields modest isolation $(<30 \mathrm{~dB})$ and high insertion loss $(>6 \mathrm{~dB})$ when compared with the waveguidebased designs [13], [14]. The remaining tunable waveguide design uses a non-magnetic substrate loaded with a ferrite bar [15]. Loaded waveguide devices are challenging to fabricate, and the placement of the ferrite can significantly alter the performance of the design. To remedy these problems, this work presents tunable full mode and half mode RWG fully ferrite filled isolators using an yttrium-iron-garnet (YIG) substrate. The full mode design requires two oppositely directly magnetic bias $H_{0}$ in the two halves of the waveguide as shown in Fig. 1(a). Such an arrangement excites a unidirectional (MSW) mode in the waveguide. A drawback of the full-mode RWG isolator is that it requires the application of two opposite bias directions. Furthermore, the performance of the isolator depends heavily on the position and abruptness of the magnetic 
bias reversal, which is assumed to occur exactly at the center of the waveguide. To mitigate this problem, a half-mode RWG isolator can be used instead, as shown in Fig. 1(b), where a perfect magnetic conductor (PMC) boundary condition is approximated along the open wall of the waveguide [16], [17]. The principle of operation of the half-mode isolator is expected to be the same as that of the full-mode isolator. Instead of using subtractive manufacturing techniques, inkjet printing is used for the isolator realization on a YIG substrate for the half-mode design. Using an additive process reduces the cost by avoiding material wastage and simplifies fabrication. The first-ever experimental demonstration of a half-mode isolator design was reported in [18]. However, the authors of that work neither proposed a theoretical model nor did they show the frequency tuning of the design. Nogouchi for the first time proposed the theory of operation of a half-mode isolator containing both a biased and an unbiased region[16], but the dispersion diagram of the resulting device is different from the one considered herein [12]. This work uses the unidirectional MSW mode rather than the resonance absorption obtained from two different modes in the half-mode isolator of [16]. In [19], the authors themselves presented a half-mode isolator design but its performance was not optimized and no theory was proposed for such a design.

In what follows, a new theoretical model is proposed that can be used to predict the center frequency and the bandwidth of an RWG ferrite isolator. Using the theory, one can determine the required magnetic field strength inside the substrate for a particular band of operation. The results obtained from the theory are compared with the measured values for both designs. The full-mode design exhibits an isolator figure of merit (IFM) of more than $65 \mathrm{~dB}$, while the half-mode design shows a peak value of $76.7 \mathrm{~dB}$, which to the author's knowledge is the highest reported value. The center frequencies of the two designs have been tuned by varying the applied magnetic field, thus showing their viability in tunable RF applications.

\section{THEORY}

Ferrites are anisotropic materials due to their response to an applied magnetic field. Solving the dominant $\mathrm{TE}^{10}$ mode inside a full-mode RWG with a bias normal to the substrate results in the following closed form equation [12],

$$
\kappa \gamma+j \mu k_{\mathrm{x}} \cot \left(a k_{\mathrm{x}}\right)=0
$$

where $\mu$ and $\kappa$ are the elements of the permeability tensor and are given by Polder's equation when the ferrite is completely saturated [20], $k_{\mathrm{x}}$ is the cut-off wave number for the direction of propagation in the $\mathrm{x}$-direction and $2 a$ is the width of the waveguide. The same equation is true for a half-mode waveguide where $a$ in itself will be the total width. The wave number $k_{\mathrm{x}}$ can be expressed as,

$$
k_{\mathrm{x}}=\sqrt{4 \pi^{2} f^{2} \varepsilon_{r} \varepsilon_{0} \mu_{e f f}+\gamma^{2}}
$$

where $\mu_{\mathrm{e}}$ is the effective permeability and can be defined by the equation,

$$
\mu_{e f f}=\frac{\mu^{2}-\kappa^{2}}{\mu}
$$

Note that (3) is the same canonical equation which is obtained for an isotropic medium with the only difference being the effective permeability of the medium. (4) can be expressed in terms of poles and zeros as,

$$
\mu_{e f f}=\mu_{0} \frac{f^{2}-f_{z}^{2}}{f^{2}-f_{p}{ }^{2}}
$$

In the above equation $f_{\mathrm{p}}$ and $f_{\mathrm{z}}$ are the poles and zeros of the function, respectively, whereas $\mu_{0}$ is the free space permeability. The zero is located at $f_{\mathrm{z}}=f_{0}+f_{\mathrm{m}}$ whereas the pole is located at $f_{p}=\sqrt{f_{0}\left(f_{0}+f_{m}\right)}$, where $f_{0}=\mu_{0} \gamma H_{0}$ is the gyromagnetic resonant frequency, $f_{\mathrm{m}}=\mu_{0} \gamma M_{s}$ is the magnetization frequency, $\gamma$ is the gyromagnetic ratio constant, $H_{0}$ is the internal magnetostatic bias field, and $M_{s}$ is the saturation magnetization. A plot of the effective permeability $\left(\mu_{\text {eff }}\right)$ as a function of the frequency for a saturated ferrite $\left(H_{0}=1350 \mathrm{Oe}, 4 \pi M_{s}=1780 \mathrm{G}\right)$ is shown in Fig. 2. The plot shows that the effective permeability has negative values in the range $f_{p}<f<f_{z}$. When this is the case, (2) admits a low loss solution in one direction only. So if a wave is propagating inside the waveguide within this band, it will be absorbed by the material, in one direction of propagation, resulting in high insertion loss while allowing transmission in the opposite direction. As a result, the waveguide can be used as an isolator within this range of frequencies. Furthermore, since $f_{p}$ and $f_{z}$ are functions of $H_{0}$ and $M_{s}$, it is possible to control the isolation band's centre frequency. It is important to explain it here that the imaginary parts of the permeability tensor elements have not been considered for the theoretical model thus assuming a lossless case to simplify the analysis. In [20], Pozar has expressed the imaginary part of $\mu_{\text {eff }}$. It will be the same here and can be used depending upon the nature of the substrate that will be determined by the linewidth $(\Delta \mathrm{H})$.

If the applied bias is varied then this negative permeability band can be tuned to control the isolation band. In this way, a tunable isolator can be realized. Defining the centre frequency of the isolation band as $f_{\mathrm{c}}=1 / 2\left(f_{\mathrm{p}}+f_{\mathrm{z}}\right)$ and the relative bandwidth as $\Omega=\left(f_{\mathrm{z}}-f_{\mathrm{p}}\right) / f_{\mathrm{c}}$, it is straightforward to show that the bandwidth is given by:

$$
\Omega=2 \frac{\sqrt{1+\zeta}-1}{\sqrt{1+\zeta}+1}
$$

where, $\zeta=\frac{M_{S}}{H_{0}}$. If a single ferrite material is considered for the design, it will fix the value of $M_{s}$; however, the value of $H_{0}$ can be varied through a large range. Thus the bandwidth and the centre frequency of the isolation band can be controlled by using a variable magnetic bias. The bandwidth $(\Omega)$ versus $\zeta$ is shown in Fig. 3, noting that the maximum bandwidth approaches $200 \%$ when the bias field $H_{0}$ is very weak. Under these conditions, the 


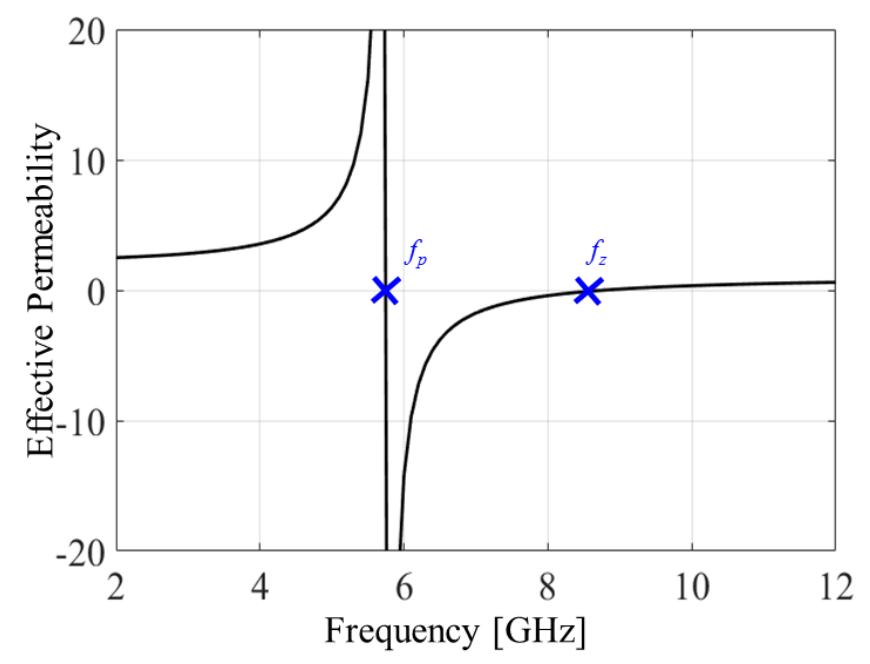

Fig. 2. Effective permeability of a ferrite substrate versus frequency $\left(H_{0}=1350 \mathrm{Oe}, 4 \pi M_{s}=1780 \mathrm{G}\right)$

design may suffer from low field losses due to the lack of saturation of the ferrite material. As a result, the ferrite may not provide good isolation performance at very weak values of $H_{0}$. If the isolation band is kept strictly above the magnetization frequency $f_{\mathrm{p}}>f_{\mathrm{m}}$ then low field losses will be prevented. However, this conservative approach to controlling low-field loss significantly reduces the available bandwidth, as shown by the dashed lines in Fig. 3, where a maximum bandwidth of only $47.2 \%$ results when $f_{\mathrm{p}}=f_{\mathrm{m}}$. Interestingly, when $f_{\mathrm{p}}=f_{\mathrm{m}}, \zeta=\varphi=$ 1.618 , the Golden Ratio. In practice, for ferrites with a low coercive field strength $H_{\mathrm{c}}$ or a high remanence $B_{\mathrm{r}}$ or $M_{\mathrm{r}}$, it is likely that magnetic saturation can be maintained at much lower magnetostatic bias, meaning that the bandwidth of the isolator can readily extend beyond $47.2 \%$ when $M_{\mathrm{s}}$ is large compared to $H_{0}$.

If the desired centre frequency of the isolator band $f_{\mathrm{c}}$ and the desired relative bandwidth $\Omega$ are known, as they would be in an application, then (6) may be used to obtain the required saturation magnetization and internal bias field:

$$
\begin{aligned}
& f_{0}=f_{\mathrm{c}} \frac{(1-\Omega / 2)^{2}}{(1+\Omega / 2)} \\
& f_{\mathrm{m}}=f_{\mathrm{c}} \frac{2 \Omega}{(1+\Omega / 2)}
\end{aligned}
$$

For example, if $f_{\mathrm{c}}=5 \mathrm{GHz}$ and $\Omega=0.4(40 \%)$, then a bias field of $H_{0}=952$ Oe $(76.2 \mathrm{kA} / \mathrm{m})$ and a ferrite magnetization of $4 \pi$ $M_{\mathrm{s}}=1189 \mathrm{G}\left(M_{\mathrm{s}}=94.6 \mathrm{kA} / \mathrm{m}\right)$ would be required. The theoretical analysis in this section applies to both the full-mode and half-mode RWG isolators.

Table I. Properties of TCI YG-1780 Substrate

\begin{tabular}{|c|c|c|c|c|}
\hline$\varepsilon_{\mathrm{r}}$ & $\Delta \mathrm{H}[\mathrm{Oe}]$ & $\tan \delta$ & $M_{s}[\mathrm{G}]$ & $H_{c}[\mathrm{Oe}]$ \\
\hline 15.0 & 30 & $<0.0002$ & 1775 & 0.5 \\
\hline
\end{tabular}

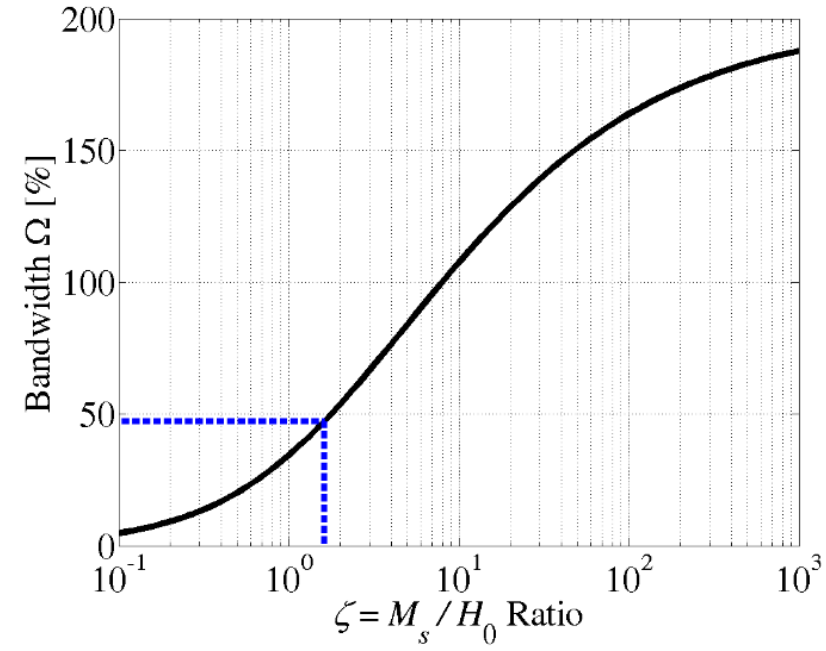

Fig. 3. Relative bandwidth of the ferrite isolator versus the magnetization-to-bias field ratio. The dashed line indicates a $47.2 \%$ bandwidth when the isolator is operated above the magnetization frequency to avoid low-field losses $\left(f_{\mathrm{p}}=f_{\mathrm{m}}, \zeta=1.618\right)$

\section{Full-Mode RWG YIG Isolator}

\section{A. Design and Analysis}

The full-mode RWG isolator measures $0.79 \times 6 \times 20 \mathrm{~mm}$ and is fabricated from a polycrystalline YIG substrate (YG-1780) provided by TCI Ceramics, the properties of which are listed in Table I. The blank ferrite substrates were metalized with $10-\mu \mathrm{m}$ thick copper with additional thin nickel and gold protection layers. Patterning of the metal is done using a milling machine. The rectangular waveguide section of the substrate is fed by demagnetized 50- $\Omega$ microstrip lines on the same ferrite substrate. The transition to rectangular waveguide is made by flaring-out the microstrip line such that it forms the upper broad wall of the RWG. The RWG width and height are equal to the cross-section of the ferrite substrate, $2 a=6 \mathrm{~mm}$ and $b=0.79$ $\mathrm{mm}$, respectively. A photograph of the YIG ferrite isolator is shown in Fig. 4.

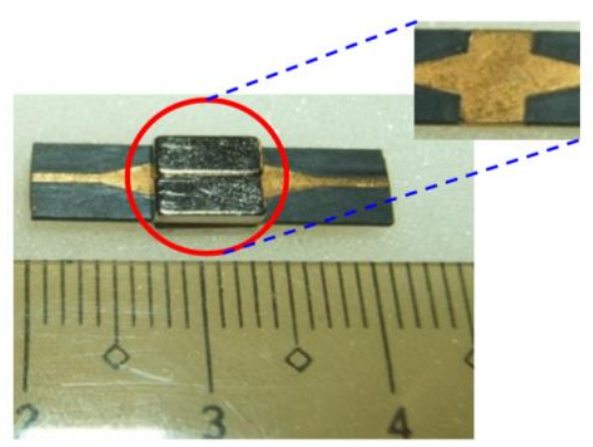

Fig. 4. Full-wave YIG isolator (bottom) and two of the magnets used for the bias (top).

The $H_{0}$ bias field is provided by neodymium magnets placed above and below the RWG portion of the circuits as shown in Fig. 5. B4201 from K\&J Magnetics Incorporation are used as the source of the magnetic fields. The magnets have dimensions 
of $0.8 \times 3.2 \times 6.4 \mathrm{~mm}$ with a fixed surface magnetic field of $2200 \mathrm{G}$. To vary the magnetic fields inside the ferrite substrate, non-magnetic spacers of height $d$ are inserted between the magnets and the substrate.

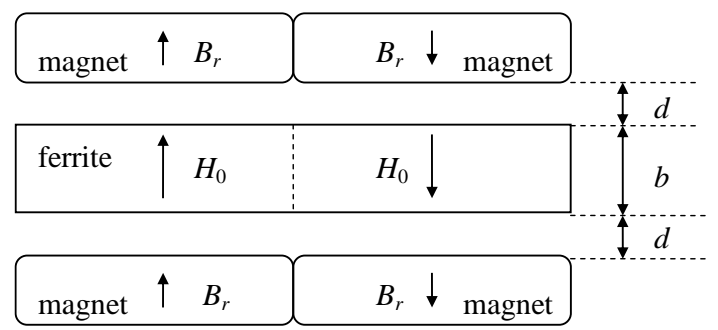

Fig. 5. Magnetization arrangement of the full-wave isolator using four permanent magnets separated by a gap $d$ from the YIG substrate of height $b$.

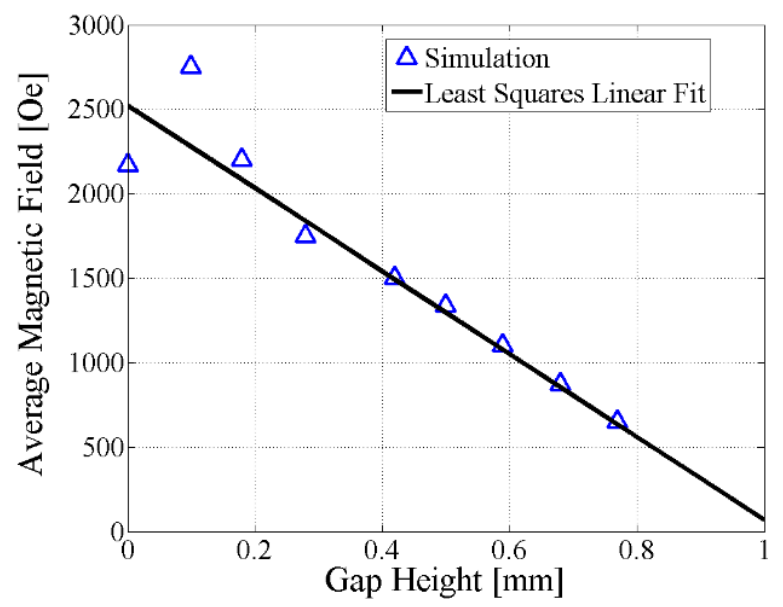

Fig. 6. Least-squares linear fit of the CST EM Studio simulated average internal vertical magnetostatic field $H_{0}$ in the YG-1780 as a function of the gap height $d$ for the antisymmetric bias (full-wave) case.

Estimates of the $H_{0}$ bias field inside the waveguide are obtained using the 3D magnetostatic field simulator CST EM Studio. The z-polarized magnetostatic fields $H_{0}$ is approximately uniform across the waveguide cross-section. Although significant $x$-polarized fields are also created near the magnets, these do not couple to the MSW mode and are of no consequence apart from weakening the $y$-polarized component of the bias field [12]. The z-polarized bias field as a function of the gap height $d$ is shown in Fig. 6, where a least-squares error linear fit was applied to the curves. For air gaps $0<d<1 \mathrm{~mm}$, the $H_{0}$ field varies from 2500 Oe to 125 Oe in the YG-1780 garnet. The data extracted from Fig. 6 can be used to predict the values of $f_{p}$ and $f_{z}$ for a particular magnetic bias strength. The results thus obtained from the theoretical analysis will be compared with the measured data in the following section.

\section{B. Measurements}

Microwave measurements of the full-wave isolator were performed using an Anritsu 3680 Universal Test Fixture (UTF). The UTF physically supports the ferrite substrate between spring-loaded jaws and performs the coaxial-to-microstrip transition. The network analyzer was calibrated using the SOLT method to the ends of the coaxial cables that connect to the UTF. Losses associated with the microstrip feed lines were removed by measuring a separate microstrip line on an identical, demagnetized ferrite substrate, from which the per-unit-length loss was obtained. The RWG portion of the isolator, located between the flared microstrip transitions as shown in Fig. 4, is 6-mm long. Measurements were taken up to $20 \mathrm{GHz}$ for gap heights $0<d<0.5 \mathrm{~mm}$. An isolation band was clearly evident in all of the measurements. The magnitude and phase response of the measured S parameters for a magnetic field strength $H_{0}$ of $\sim 1350$ Oe (corresponding to a distance of $0.5 \mathrm{~mm}$ ) are shown in Fig. 7 and Fig. 8, respectively. It is important to explain that how the corner frequencies of the isolation band are identified. Theoretically, the unidirectional edge-guided mode exists in the range $f_{p}<f<f_{z}$ under lossless conditions. Care must be taken when attempting to identify the band edges of this mode based on the measured data. Although insertion loss offers a clue as to the location of the band edges, it is also subjected to impedance mismatch (especially near the band edges where the modes change) which masks the location of the band edge. For the pole frequency, $f_{p}$ the amplitude plot can be considered, i.e. Fig. 7. A dip can be seen in the forward mode's transmission that can reliably estimate $f_{p}$, which is $5.48 \mathrm{GHz}$. For the zero frequency $f_{z}$, the phase response of the isolator needs to be considered, as explained in [12]. Above the frequency $f_{z}$, the reverse mode (high attenuation) becomes a regular $\mathrm{TE}^{10} \mathrm{RWG}$ mode for which the phase constant approaches zero (or even becomes a backward wave) at $f_{z}$. As such, we expect to see a phase inflexion point in the phase of the reverse mode to signal the $f_{z}$ frequency. This concept is illustrated in Fig. 8 where $f_{z}$ comes out to be 8.7 $\mathrm{GHz}$. Using the bias strength of $1350 \mathrm{Oe}$, the theoretical model gives the corner frequencies $\left(f_{p}, f_{z}\right)$ of $5.4 \mathrm{GHz}$ and $8.7 \mathrm{GHz}$, respectively. These values nicely align with the measured results thus verifying the theoretical model. In this way the pole and zero frequency points are recorded in the measurements for different bias strengths. A comparison between the theory and the measurements of the full-mode isolator design is shown in Fig. 9. A good agreement is achieved between the two results.

The measured isolation figure of merit (IFM), defined as $\left|S_{21}\right|_{\mathrm{dB}}-\left|S_{12}\right|_{\mathrm{dB}}$, for this bias value is shown versus frequency in Fig. 10. The maximum value of IFM is measured to be $65.1 \mathrm{~dB}$. For this measurement, the minimum value of insertion loss is $1.3 \mathrm{~dB}$. The IFM generally decreased as the isolator's centre frequency was increased by increasing the $H_{0}$ field, although IFMs of greater than $20 \mathrm{~dB}$ were realized up to and including centre frequency of around $11 \mathrm{GHz}(10.7 \mathrm{GHz}$ to be precise) as shown in Fig. 11. The measured centre frequency of the isolator is tuned from $7 \mathrm{GHz}$ to $10.7 \mathrm{GHz}$ by varying the bias fields from 1350 Oe to 2500 Oe. Consequently, the measured bandwidth varies from $44.7 \%$ to $28.7 \%$, changing the $f_{\mathrm{p}}$ and $f_{\mathrm{z}}$ of the isolator from $5.48 \mathrm{GHz}$ to $9.13 \mathrm{GHz}$ and $8.7 \mathrm{GHz}$ to 12.2 $\mathrm{GHz}$, respectively. During this tuning range the isolator maintains acceptable impedance match performance with insertion loss degrading at stronger bias levels. Up to 2000 Oe of bias field the insertion loss is satisfactory. Depending on the 
application, the maximum bias strength can be chosen to keep the insertion loss in a reasonable range. The results thus obtained verify the concept of tuning the centre frequency and the bandwidth of a RWG ferrite isolator by varying the magnetostatic bias across it.

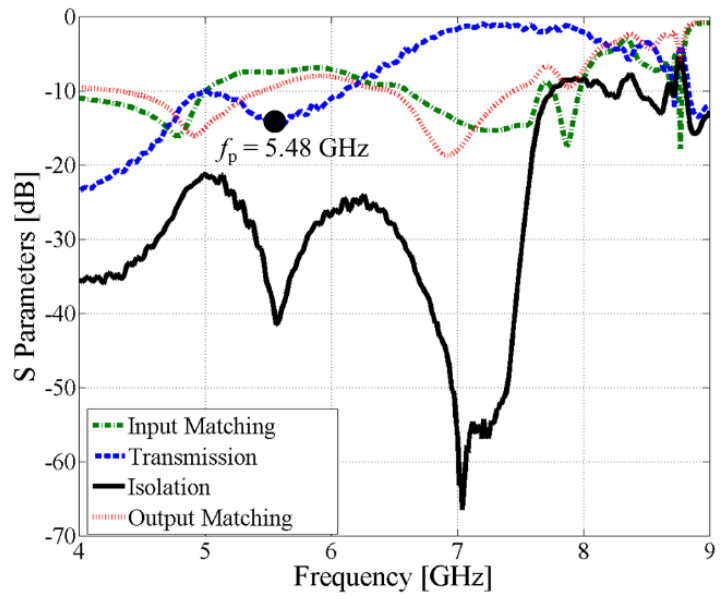

Fig. 7. Measured S parameters of the full-mode isolator for an $H_{0}$ of $1350 \mathrm{Oe}$

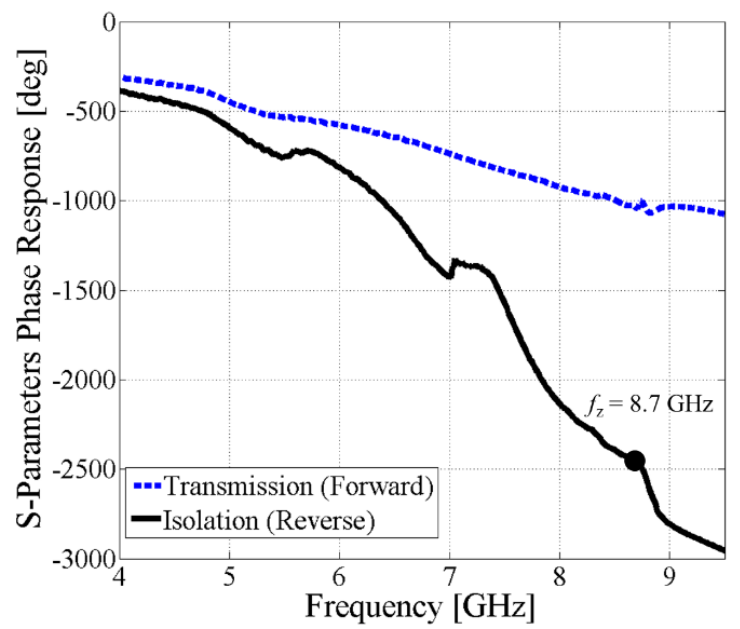

Fig. 8. Phase response of the full-mode isolator for an $H_{0}$ of 1350 Oe

\section{HALF-MODE RWG YIG IsOLATOR}

\section{A. Design and Fabrication}

Using the same theory as presented in Section II, a half-mode RWG isolator is designed on the same substrate as the full-mode design. The dimensions for this waveguide are $4.8 \mathrm{~mm} \times 0.4 \mathrm{~mm}$ with a length of $10 \mathrm{~mm}$, as shown in Fig. 12. The RWG to microstrip transition has a length of $7 \mathrm{~mm}$ which results in a total device length of $24 \mathrm{~mm}$. An inkjet printing device, the Dimatix 2831 materials printer, is used for printing the metallic layers on the YIG substrate using a silver-organo-complex (SOC) based ink [21]. The conductivity of the printed metal can be increased by printing multiple layers of ink. In addition, this will also help in improving the insertion loss performance due to the skin effect at microwave frequencies. Six layers of the metallic ink are printed to realize the half-mode isolator. After printing, the

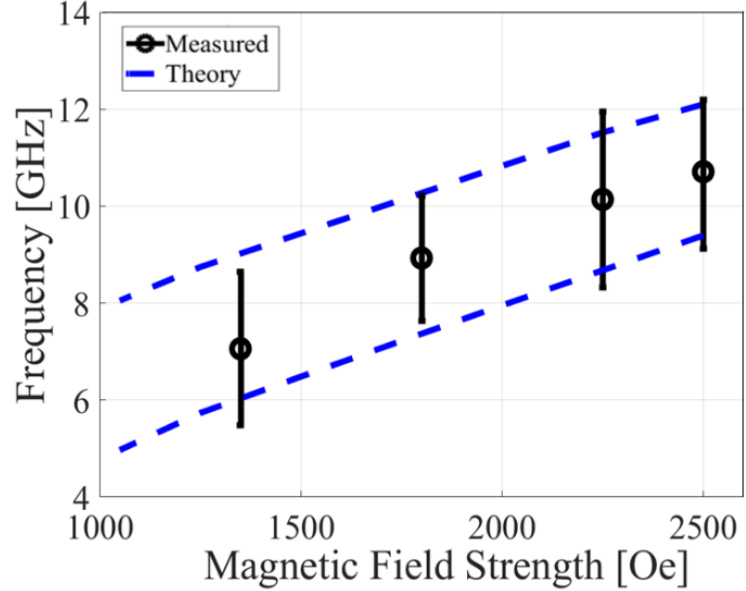

Fig. 9. Theoretical vs. measured bandwidth of full-mode isolator vs. the magnetic field strength

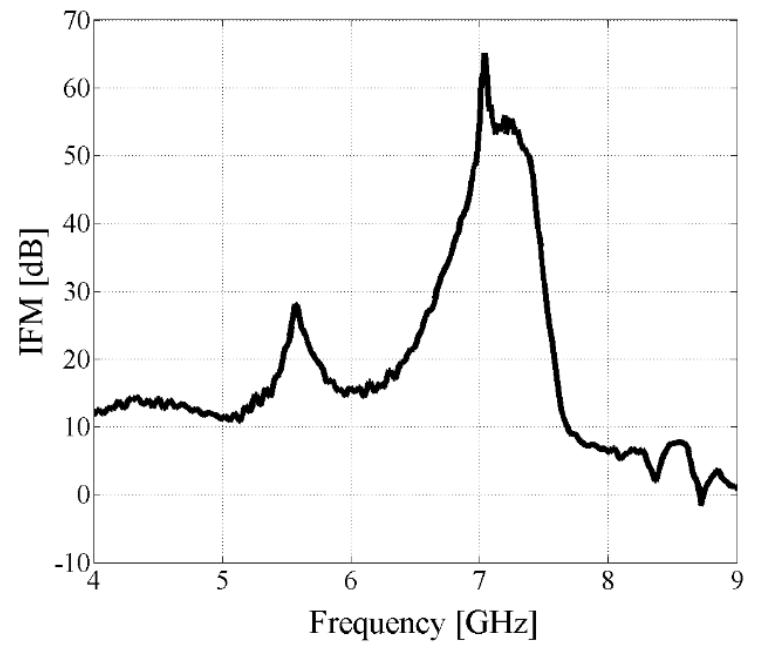

Fig. 10. Measured IFM of the full-mode isolator for an $H_{0}$ of 1350 Oe

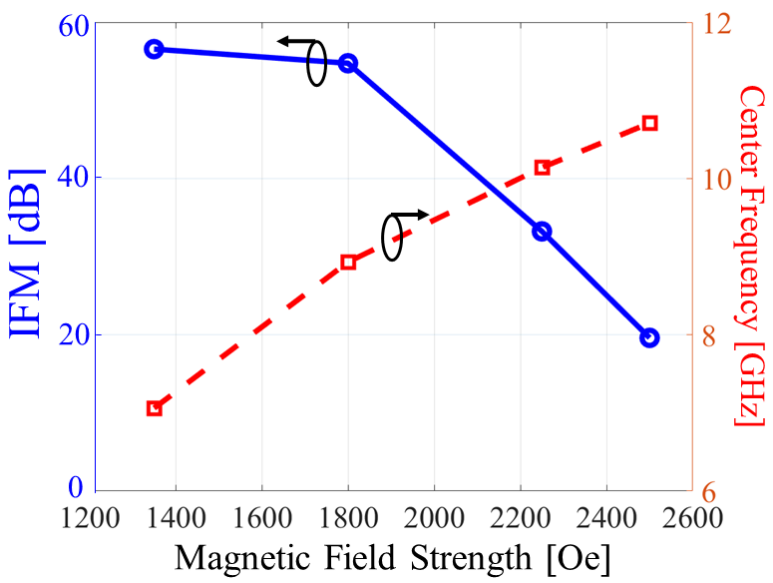

Fig. 11. IFM and center frequency vs. magnetic field strength of the full-mode isolator

substrate is heated to $80^{\circ} \mathrm{C}$ (using infrared heating) for $5 \mathrm{~min}$. The photograph of the prototype isolator with the SMA connectors is shown in Fig. 13. 


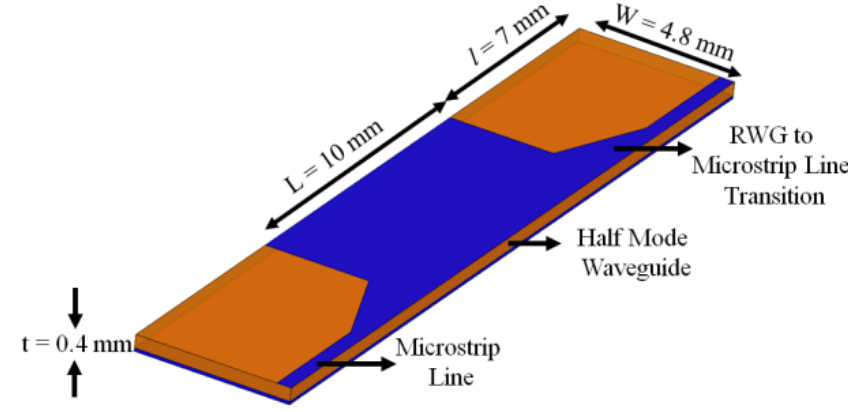

Fig. 12. Half-mode isolator design

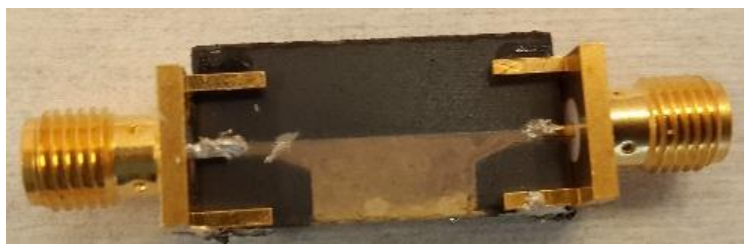

Fig. 13. Fabricated half-mode isolator

\section{B. Magnetostatic Simulations and Measurements}

Since this geometry of the half-mode isolator requires only a unidirectional magnetic bias field, conventional electromagnet can be used for its measurements instead of using permanent magnets. Thus, the design itself provides a simpler strategy for the measurements of its S-parameters. The applied bias magnetic field of the electromagnet is varied from 0 Oe to 3500 Oe. To determine the magnetic field strength inside the substrate, magnetostatic simulations are again carried out in CST EM Studio. The fields generated by an electromagnet are generally uniform. However, to have an exact estimate of the field strength inside the substrate, the demagnetization fields must also be assessed through simulation. The z-polarized magnetic field strength inside the substrate was recorded and averaged for different applied field strengths. This strategy is similar to the one used by the authors in the previous reported ferrite based microwave designs [22]-[24]. The relationship between the applied and internal magnetic field strength for the half-mode isolator is shown in Fig. 14. The information obtained from these simulations are used to compare the measured tuning range of the isolator with the theoretical model.

When subjected to an external bias strength of $1500 \mathrm{Oe}$ (internal bias strength of $322.1 \mathrm{Oe}$ ), the half-mode isolator shows a transmission coefficient of $3.5 \mathrm{~dB}$ with an isolation of $58.2 \mathrm{~dB}$, as shown in Fig. 15. The center frequency of the isolator is $4.4 \mathrm{GHz}$ with the pole and zero frequencies of 3.1 $\mathrm{GHz}$ and $5.7 \mathrm{GHz}$, respectively. The pole and zero frequency of the half-mode design are extracted in the same manner as explained in Section III-B for the full-mode isolator. Excluding the loss from the RWG to micrsotrip transition results in an insertion loss of $1.45 \mathrm{~dB}$ which is comparable to the full-mode design. The slightly increased value of the insertion loss can be attributed to the conductivity of the silver ink which will be less than the conductivity of the conductor used in the full-wave isolator. Another important observation in this measurement is the increased transmission loss at $3.8 \mathrm{GHz}$ and $4.7 \mathrm{GHz}$. Losses are incurred at these frequencies because they are lower than the magnetization frequency of the YIG substrate $\left(f_{m}=4.98\right.$ $\mathrm{GHz}$ ). Although the waveguide part of the isolator is saturated, the feed lines are operated in the demagnetized state. Therefore, the losses below $f_{m}$ are quite evident even after saturating the isolator region. For frequencies above $5 \mathrm{GHz}$, the transmission loss is on the higher side due to the mismatch at the input and the output. Since, the RWG-to-microstrip transition is a narrow band solution, the matching is not very good above $5 \mathrm{GHz}$. Nonetheless a good isolation performance can be clearly observed from the measured results, as can be seen from the isolation figure of merit, in Fig. 16. The maximum value of IFM is measured to be $54.6 \mathrm{~dB}$ for an internal bias strength of 322.1 Oe.

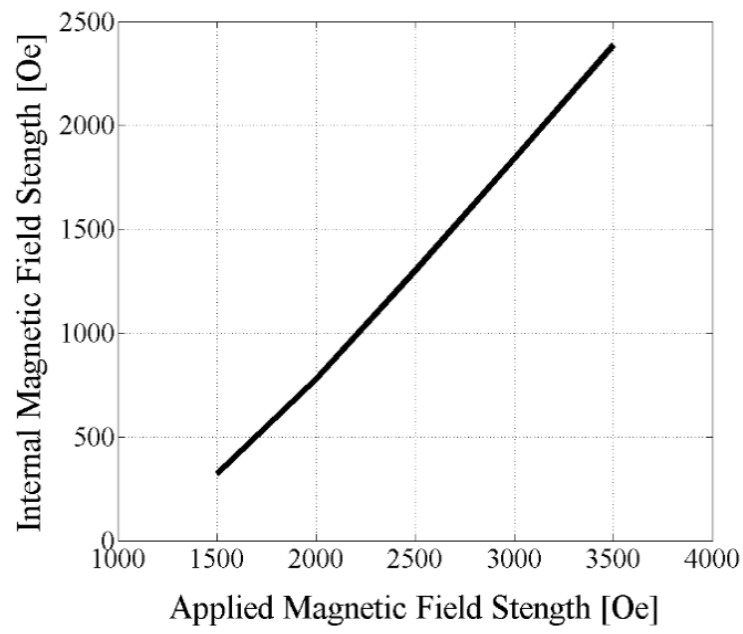

Fig. 14. Internal vs. applied magnetic field strength from the electromagnet

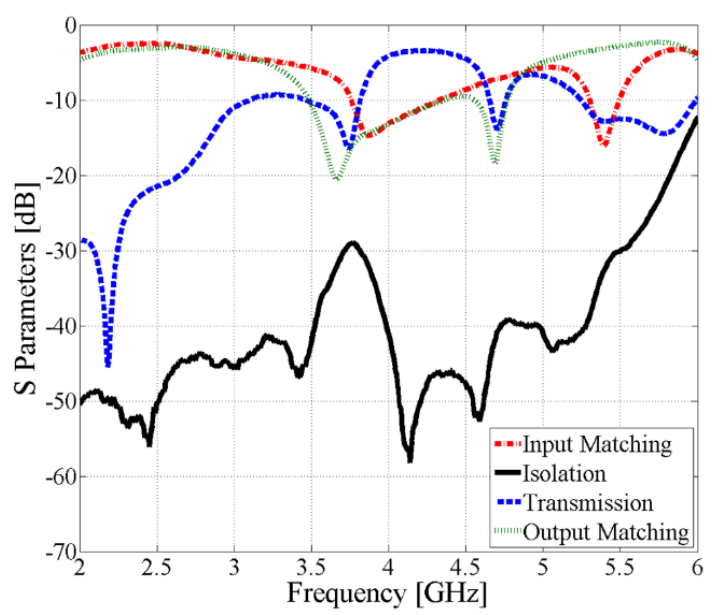

Fig. 15. Measured S Parameters of the half-mode isolator design for an $H_{0}$ of $322.1 \mathrm{Oe}$ 


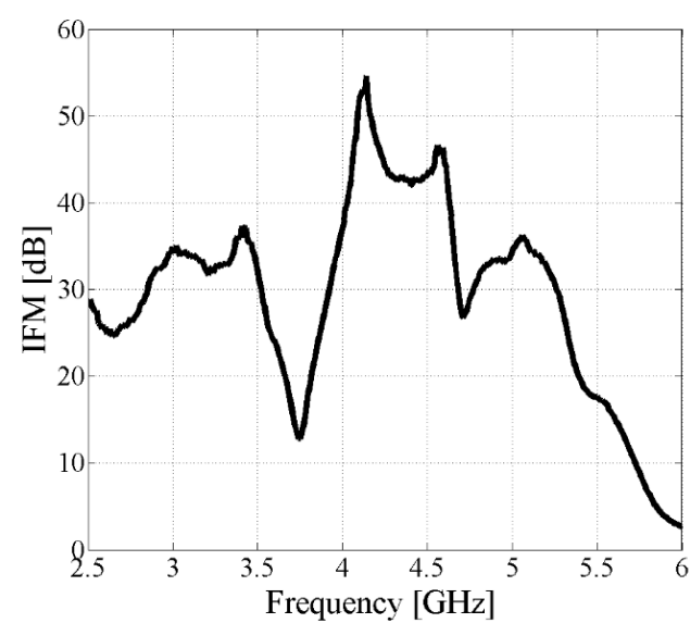

Fig. 16. Measured IFM of the isolator for an $H_{0}$ of $322.1 \mathrm{Oe}$

The measurements of the biased waveguide show good isolation performance at 1500 Oe (internal field strength of $322.1 \mathrm{Oe}$ ). Now the applied magnetostatic field is varied across the device to tune the isolation band. The applied bias field is varied from 1500 Oe to 3500 Oe. The internal magnetic field strength for these values can be obtained from Fig. 14. The use of the electromagnet makes it easier to vary the strength of the magnetic bias as opposed to the case of the full-mode design where permanent magnets are used. Thus, the authors are able to reduce the strength of the magnetic field down to few hundred Oe, resulting in a larger tuning range unlike the fullmode design. For each magnetic field value, the pole $\left(f_{\mathrm{p}}\right)$ and zero $\left(f_{\mathrm{z}}\right)$ frequencies of the device are recorded. The tuning of $f_{\mathrm{p}}$ and $f_{\mathrm{z}}$ versus the internal magnetostatic bias field strength is shown in Fig. 17. Assuming the two frequencies to be the egdes of the isolation band, the center frequency of the isolator is calculated and varies from $4.4 \mathrm{GHz}$ to $9.9 \mathrm{GHz}$. The bandwidth of the half-mode design is tuned from $58.8 \%$ to $30.9 \%$. Compared to theory, the measured bandwidth of the half-mode design is in good agreement. The variation of the maximum IFM and the center frequency with the internal bias strength is shown in Fig. 18. The IFM values generally remain above 40 $\mathrm{dB}$ with the maximum value of $76.7 \mathrm{~dB}$ observed at $7.5 \mathrm{GHz}$ for a bias field strength of $1840 \mathrm{Oe}$. Increasing the applied bias beyond $3500 \mathrm{Oe}$ (internal bias strength of $2386 \mathrm{Oe}$ ) can further tune the isolation band but with a higher insertion loss due to poor input and output matching. These measurements provide a proof-of-concept that by varying the magnetic field across the half-mode waveguide isolator, the band of isolation can be tuned. In future, the authors recommend the study of these class of designs in multilayer technology such as Ferrite Low Temperature Cofired Ceramic (LTCC) [22]-[25].

\section{CONCLUSION}

Two YIG RWG isolator designs are reported in this work. A half-mode inkjet printed YIG isolator is reported that demonstrates frequency tuning. A novel analytical model has been provided to predict the center frequency and the bandwidth of the full-mode and half-mode isolators. Useful design equations have also been developed to describe the tuning of the isolators. A good agreement between the theory and measurements has been achieved, thus validating the proposed analytical model. The measured results show that IFM values as high as $65 \mathrm{~dB}$ and $76.7 \mathrm{~dB}$ can be achieved for the full-wave and half-wave isolators, respectively. The center frequency of the isolators has been tuned using the variable applied magnetic field. For the full-mode design, the center frequency is tuned from $7 \mathrm{GHz}$ to $10.7 \mathrm{GHz}$, while for the halfmode design the tuning changes the center frequency from 4.4 $\mathrm{GHz}$ to $9.9 \mathrm{GHz}$. The bandwidths of the two designs are varied from $44.7 \%$ to $28.7 \%$ and $58.8 \%$ to $30.9 \%$, respectively. These designs show that the center frequency and the bandwidth of a ferrite isolator can be reconfigured depending upon system requirements, making them a viable option for tunable high frequency applications.

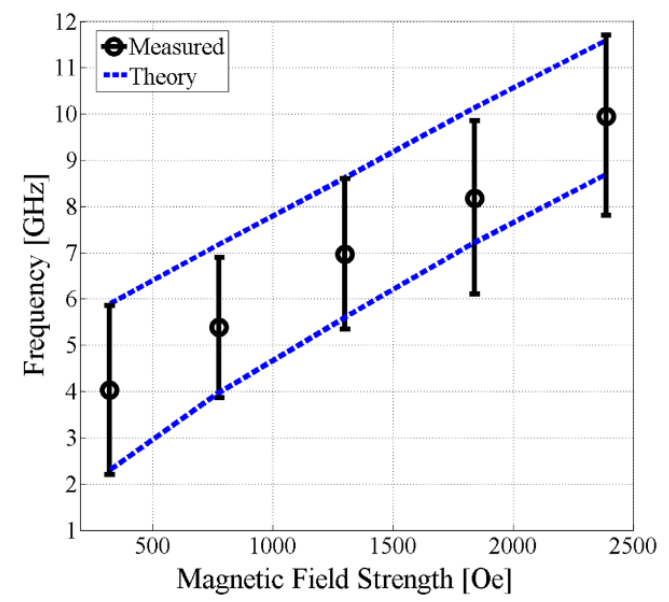

Fig. 17. Tuning of isolation bandwidth of the half-mode isolator design

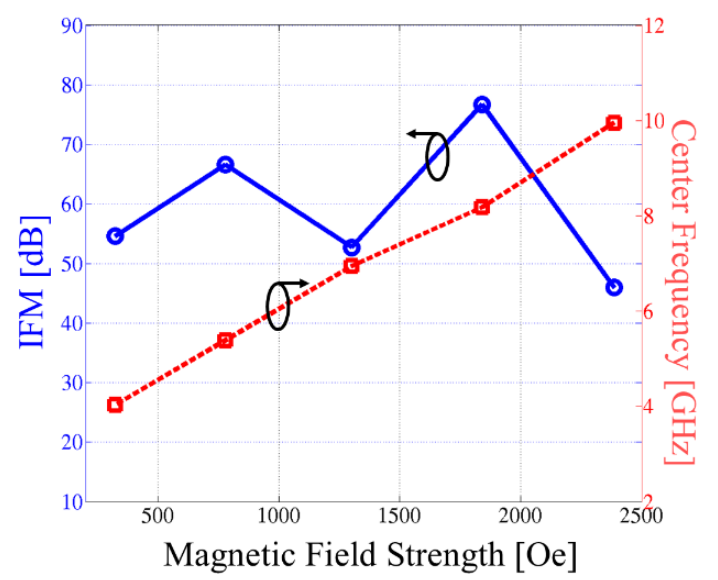

Fig. 18. IFM and center frequency vs. magnetic field strength of the half-mode isolator

\section{REFERENCES}

[1] F. Okada, K. Ohwi, and Y. Yokochi, "YIG resonator circuit with isolator property and its application to a Gunn diode oscillator," in IEEE Transactions on Microwave Theory and Techniques, vol. 26, no. 12, pp. 1035-1039, Dec 1978. 
[2] M. van Heijningen, et. al., "L-band AlGaN/GaN power amplifier with protection against load mismatch," IEEE European Microwave Integrated Circuit Conference, pp. 408-411, 2013.

[3] J. F. Chang, J. C. Kao, Y. H. Lin, and H. Wang, "Design and analysis of 24-GHz active isolator and quasi-circulator," in IEEE Transactions on Microwave Theory and Techniques, vol. 63, no. 8, pp. 2638-2649, 2015.

[4] D. G. Haigh, "Wideband active microwave isolators using GaAs MMIC technology," in IEE Proceedings - Microwaves, Antennas and Propagation, vol. 143, no. 2, pp. 179-183, 1996.

[5] P. Pursula, I. Marttila, and K. Nummila, "Wideband adaptive isolator for UHF RFID reader," in Electronics Letters, vol. 45, no. 12, pp. 636-637, June 42009.

[6] J. Helszajn and M. Mckay, "Circular polarisation in a double-ridge waveguide," IEE Proceedings Microwaves, Antennas and Propagation, vol. 152, no. 1, pp. 25-30, 2005.

[7] G. L. Matthaei, E. M. T. Jones, and S. B. Cohn, "A nonreciprocal, TEMmode structure for wide-band gyrator and isolator applications," IRE Transactions on Microwave Theory and Techniques, vol.7, no. 4, pp. 453460, 1959.

[8] F. Fesharaki, C. Akyel, and K. Wu, "Broadband substrate integrated waveguide edge-guided mode isolator," Electronics Letters, vo1.49, no.4, pp. 269-271, 2013.

[9] A. Beyer and I. Wolff, "Design of a millimetre-wave ferrite isolator at 28.5 GHz," IEE Proceedings $H$ - Microwaves, Antennas and Propagation, vol. 132, no. 4, pp. 244-250, 1985.

[10] M. F. Farooqui, A. Nafe and A. Shamim, "Inkjet printed ferrite-filled rectangular waveguide X-band isolator," 2014 IEEE MTT-S International Microwave Symposium (IMS2014), pp. 1-4, 2014.

[11] M. E. Hines, "Reciprocal and nonreciprocal model of propagation in ferrite stripling and micrsotrip devices," IEEE Transactions on Microwave Theory and Techniques, vol. 19, no. 5, pp. 442-451, 1971.

[12] C. K. Seewald and J. R. Bray, "Ferrite-filled antisymmetrically biased rectangular waveguide isolator using magnetostatic surface wave modes," IEEE Trans. Microw. Theory Tech., vol. 58, pp. 1493-1501, June 2010.

[13] S. Capraro et al., "Feasibility of an integrated self-biased coplanar isolator with barium ferrite films," in IEEE Transactions on Components and Packaging Technologies, vol. 30, no. 3, pp. 411-415, 2007.

[14] S. Subbiah and A. Alphones, "Tunable isolator using a coupled microstrip line with an obliquely magnetised YIG substrate," IEE Proceedings Microwaves, Antennas and Propagation, vol. 150, no. 4, pp. 219-222, 2003.

[15] Y. J. Cheng, Q. D. Huang, Y. R. Wang and J. L. W. Li, "Narrowband substrate integrated waveguide isolators," IEEE Microwave and Wireless Components Letters, vol. 24, no. 10, pp. 698-700, 2014.

[16] T. Noguchi, "New edge-guided mode isolator using ferromagnetic resonance absorption," IEEE Transactions on Microwave Theory and Techniques, vol. 25, pp. 100-106, Feb. 1977.

[17] F. A. Ghaffar and A. Shamim, "A partially magnetized ferrite LTCC based SIW phase shifter for phased array applications", IEEE Transactions on Magnetics, vol.51, no.6, pp.1-8, 2015.

[18] K. Araki, T. Koyama, and Y. Naito, "A new type of isolator using the edge-guided mode," IEEE Trans. Microwave Theory and Techniques, vol. MTT-23, no. 3, p. 321, 1975 .

[19] F. A. Ghaffar, M. Vaseem, J. R. Bray, and A. Shamim, "A half mode inkjet printed tunable ferrite isolator," IEEE International Microwave Symposium (IMS), pp. 289-291, 2017.

[20] D. M. Pozar, Microwave Engineering, $3^{\text {rd }}$ ed. New York: Wiley, 1993, ch. 9.

[21] G. McKerricher, M. Vaseem, and A. Shamim "Fully Inkjet Printed RF Inductors and Capacitors using Polymer Dielectric and Silver Conductive Ink with Through Vias", IEEE Transactions on Electron Devices (TED), vol.62, no.3, pp.1002-1009, 2015.

[22] F. A. Ghaffar, J. Bray, and A. Shamim, "Theory and Design of a Tunable Antenna on a Partially Magnetized Ferrite Substrate", IEEE Transactions on Antennas and Propagation, vol. 62, no. 3, pp. 1238-1245, 2014.

[23] F. A. Ghaffar and A. Shamim, "A Ferrite LTCC Based Dual Purpose Helical Antenna Providing Bias for Tunability," IEEE Antennas and Wireless Propagation Letters, vol. 14, pp. 831-834, 2015.
[24] A. Nafe, F. A. Ghaffar, M. F. Farooqui and A. Shamim, "A Ferrite LTCCBased Monolithic SIW Phased Antenna Array," in IEEE Transactions on Antennas and Propagation, vol. 65, no. 1, pp. 196-205, 2017.

[25] F. A. Ghaffar, A. Shamim, and K. N. Salama, "Design and comparison of LTCC based fractal antennas," Proceedings of URSI General Assembly Science Symposium, 2011.

Farhan A. Ghaffar received his B.E. degree in electronics engineering from the NED University of Engineering and Technology, Karachi, Pakistan, in 2007, and the M.S. and PhD degrees in electrical engineering from the King Abdullah University of Science and Technology (KAUST), Thuwal, Saudi Arabia, in 2010 and 2016 respectively. He is currently working as a Postdoctoral Fellow and Instructor at University of Ontario Institute of Technology (UOIT), Oshawa, ON, Canada.

He was an Assistant Manager with Pakistan Space and Upper Atmosphere Research Commission (SUPARCO), Karachi, from 2008 to 2009, before joining KAUST. He was a Visiting Researcher with Carleton University, Ottawa, ON, Canada, and Royal Military College, Kingston, ON, Canada, in 2010 and 2012, respectively. Dr. Ghaffar is an author/co-author on 35 international publications which include 13 peer-reviewed journal papers. He is a co-inventor on 3 international patents. He has been the recipient of Academic Excellence Award at KAUST in 2013 and 2015, secured Honorary Mention in the First Ever 3MT Competition at International Microwave Symposium (IMS) and was awarded best performance award at SUPARCO in 2009. His current research interests include the design of system-on-package and system-on-a-chip-based antennas, radio frequency integrated circuits, flexible microwave passive components and ferrite low temperature co-fired ceramic-based tunable antennas and passives.

Joey R. Bray (S'96-M'04) received the B.A.Sc. and M.A.Sc. degrees in electrical engineering from the University of Ottawa, Ottawa, ON, Canada, in 1995 and 1998, respectively, and the Ph.D. degree in electrical engineering from Carleton University, Ottawa, ON, Canada, in 2003.

In 2003, he joined the Department of Electrical and Computer Engineering, Royal Military College of Canada, Kingston, ON, Canada, where he is currently an Associate Professor and the Lead Investigator of the Electronic Warfare, Antennas and Radar research group. From 2001 to 2002, he was a Visiting Researcher at Valtion Teknillinen Tutkimuskeskus (VTT) Electronics, Oulu, Finland. During his 2009-10 sabbatical leave, he was a Visiting Research Associate at the School of Electrical and Electronic Engineering, University of Manchester. His research interests include nonreciprocal microwave devices, radar, electromagnetic compatibility, and electronic warfare.

Dr. Bray is a licensed professional engineer in the Province of Ontario.

Mohammad Vaseem received his M.Sc. in Physical Chemistry from Aligarh Muslim University (AMU), India, in 2005 and Ph.D. in Chemical Engineering in Feb 2011 from Chonbuk National University, South Korea. He worked as a Post-Doctoral Fellow in World Class University (WCU) project and Brain Korea 21 project, Chonbuk National University, South Korea during 20112014. He also worked as Post-Doctoral Fellow in Electrical Engineering Program at KAUST during 2015-2018. Currently, he is a Research Scientist at KAUST. He is an author/co-author of over 45 international publications, an inventor on 3 patents and has over 80 research paper presentation in several national and international conferences. Dr. Vaseem current research is based on ink-development for metal, dielectric, oxide semiconductor, magnetic and several other functional materials and its inkjet printing for the fabrication of RF electronics, transistors, sensors and so on.

Langis Roy received the B.A.Sc. degree in electrical engineering from the University of Waterloo, Waterloo, ON, Canada, and the M.Eng. and Ph.D. Degrees in electrical engineering from Carleton University, Ottawa, ON, Canada. From 2005 to 2006, he was a Visiting Professor with the VTT Micromodules Research Center, Oulu, Finland, and an Invited Professor with the INSA Electrical and Computer Engineering Department, Toulouse, France. From 2009 to 2010, he was an Invited Researcher with IETR/Université de Rennes, Rennes, France. He has been a Faculty Member with the Department of Electrical and Computer Engineering, University of Ottawa, and also with the Department of Electronics, Carleton University. He is currently a Professor of electrical engineering and the Dean of Graduate Studies with the University of Ontario Institute of Technology, Oshawa, ON, Canada. He has coauthored 
over 100 scientific papers and holds three patents on system-on-package designs. His current research interests include microwave electronics, highperformance electronic circuit packaging, integrated active antennas, reconfigurable microwave components, wireless sensors, and aerospace/automotive applications, now extending to terahertz biosensing and wireless power harvesting.

Atif Shamim (SM'13) received the M.Sc. and the Ph.D. degrees in electrical engineering from Carleton University, Ottawa, ON, Canada, in 2004 and2009, respectively. From 2007 to 2009, he was a Natural Sciences and Engineering Research Council (NSERC) Alexander Graham Bell Graduate Scholar with Carleton University. From 2009 to 2010, he was an NSERC Post-Doctoral Fellow with the Royal Military College Canada, Kingston, ON, and the King Abdullah University of Science and Technology (KAUST), Thuwal, Saudi Arabia. In 2010, he joined the Electrical Engineering Pro-gram with KAUST, where he is currently an Associate Professor and a Principal Investigator with the IMPACT Lab, KAUST. He was an Invited Researcher with the VTT Micromodules Research Center, Oulu, Finland, in 2006. He has authored/coauthored over 180 international publications and an inventor on 20 patents. His current research interests include innovative antenna designs and their integration strategies with circuits and sensors for flexible and wearable wireless sensing systems through a combination of CMOS and additive manufacturing technologies. Dr. Shamim was a recipient of the Best Paper Prize from the European Microwave Association Conference in 2008 and the Ottawa Centre of Research Innovation (OCRI) Researcher of the Year 2008 Award in Canada, the ITAC SMC Award from the Canadian Microelectronics Corporation EXPO for wireless dosimeter in 2007, and the Best Paper Prize (third position) in IEEE IMS 2016 and in IEEE MECAP 2016 (first position). He was also a recipient of numerous business-related awards, including the first prize in Canada's national business plan competition and was selected for OCRI Entrepreneur of the Year Award in 2010. He serves on the Editorial Board for the IEEE Transactions on Antennas and Propagation. 